\title{
A Model for Window Based Flow Control Packet-Switched Networks
}

\author{
by \\ Xiaowei Yang \\ Submitted to the Department of Electrical Engineering and Computer \\ Science \\ in partial fulfillment of the requirements for the degree of \\ Master of Science \\ at the \\ MASSACHUSETTS INSTITUTE OF TECHNOLOGY \\ March 1998 \\ (C) Massachusetts Institute of Technology 1998. All rights reserved.
}

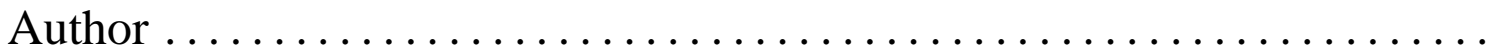

Department of Electrical Engineering and Computer Science March 6th, 1998

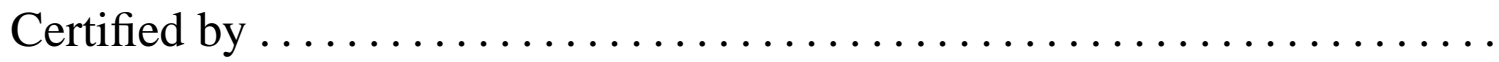

Dr. David D. Clark

Senior Research Scientist, Laboratory for Computer Science Thesis Supervisor

Accepted by

Arthur C. Smith Chairman, Departmental Committee on Graduate Students 



\title{
A Model for Window Based Flow Control Packet-Switched Networks
}

\author{
by \\ Xiaowei Yang \\ Submitted to the Department of Electrical Engineering and Computer Science \\ on March 6th, 1998, in partial fulfillment of the \\ requirements for the degree of \\ Master of Science
}

\begin{abstract}
Recently, networks have increased rapidly both in scale and speed. Problems related to the control and management are of increasing interest. However, there is no satisfactory tool to study the behavior of such networks. The traditional event driven simulation is slow when the network speed is high. The time driven simulation is against the nature of packet-switched networks. As Transmission Control Protocol (TCP) is the most widely used transport layer protocol, and it uses window based flow control mechanism, classic queuing theories involving Markov Chain assumptions are not applicable.

This thesis develops a model for the window based flow control packet-switched networks. The model attempts to provide a way to study large and high speed networks using TCP. In this thesis, we discuss in detail the construction, implementation and application of the model. This thesis also compares the results obtained from the model with those from the packet by packet event driven simulation. The comparison shows the model is correctly modeling the networks.
\end{abstract}

Thesis Supervisor: Dr. David D. Clark

Title: Senior Research Scientist, Laboratory for Computer Science 



\section{Acknowledgments}

I would like to thank my thesis advisor, Dave Clark, for his advice and support. I appreciate his kindness to give me a chance to study in the field of networking. I also appreciate his intuition and patience which helped me go through this project. I thank Karen Sollins and John Wroclawski for their help whenever I ran into problems.

I thank the system administrator of ANA group, Dorothy Curtis, who spent a lot of time in setting up my computers, installing software I need and answering all my questions about UNIX.

I thank Prof. Robert Gallager and Dr. Balaji Prabhakar, who are lecturers of Data Communication Networks. Not only has their class greatly enriched my knowledge of networking but also the advice I got from them helped me gain some insight into this project.

I thank my friends Li-wei Lehman, Dina Katabi and my former officemate Ulana Legedza, for their generous share of knowledge and their encouragement. I also thank Prof. Lixia Zhang for her guidance through emails and her kindness to help me.

I thank the people in the ANA group: Rob Cheng, Wenjia Fang, Lewis Girod, Mark Handley, Daniel Lee, Atanu Mukherjee, Alex C. Snoeren, Elliot Schwartz, Rena Yang. They are always valuable resources to find an answer to any question.

I thank my advisor of my undergraduate study at Tsinghua University, Deyun Lin. Without his recommendation, I may not have the chance to come to MIT.

I thank my family in China. Though they are far alway from me, the telephone conversations with them and their letters give me the strength to overcome all difficulties I face.

Finally, but by no means less important, I sincerely thank Daniel Jiang, for his dedicated 
love and care. 


\section{Contents}

1 Introduction $\quad 13$

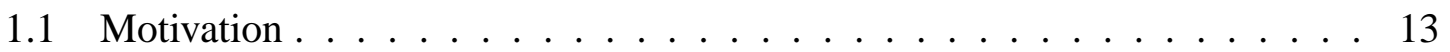

1.2 Organization of this Thesis . . . . . . . . . . . . . . 14

2 Related Work $\quad 15$

2.1 Simulation Methods . . . . . . . . . . . . . . . . . 15

2.2 Queuing Theories ........................ 17

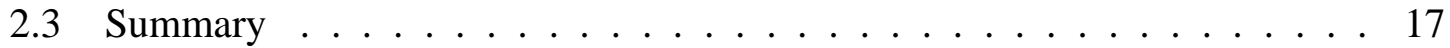

3 Model Construction $\quad 19$

3.1 End-to-end Window Based Flow Control . . . . . . . . . . . . . . . . . . . 19

3.2 Basic Assumptions . . . . . . . . . . . . . . . . 20

3.3 The Model . . . . . . . . . . . . . . . . . . . . 21

4 Algorithm $\quad 27$

4.1 The Difficult Point . . . . . . . . . . . . . . . . . 28

4.2 How to Find a Solution for the Model . . . . . . . . . . . . . . 30

4.2 .1 Ideas ............................ 30

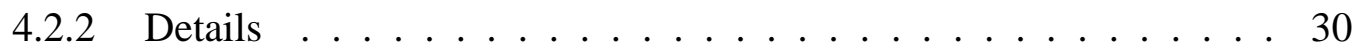

4.2.3 Another Perspective of the Algorithm . . . . . . . . . . . . 33

4.3 Short Discussion About the Implementation . . . . . . . . . . . . . 35

5 Implementation Issues and Testing Results 37

5.1 How to Reduce the Number of Iterations . . . . . . . . . . . . . . . 37 
5.2 How to Solve the Equations in $E(\alpha) \ldots \ldots \ldots \ldots$

5.3 Performance Analysis . . . . . . . . . . . . . . . . . . 41

5.4 Results Comparison with those of $n s \ldots \ldots \ldots \ldots$. . . . . . 42

5.4.1 A Brief Introduction of a Test Net . . . . . . . . . . . . 42

5.4 .2 Results . . . . . . . . . . . . . . . . . . 42

6 Application Results 45

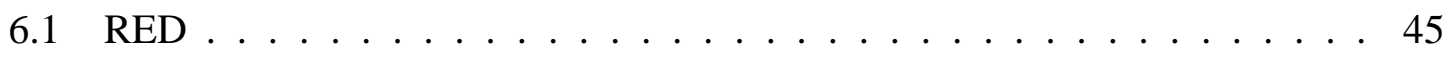

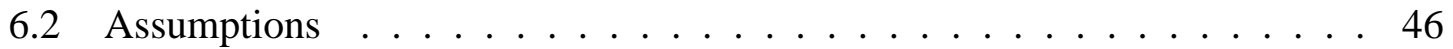

6.3 The Event Driven Simulation . . . . . . . . . . . . . . . 46

6.3.1 The Simulating Method _ . . . . . . . . . . . . . . 46

6.3 .2 Results . . . . . . . . . . . . . . . . 48

6.4 The Time Driven Simulation $\ldots \ldots \ldots$

6.4 .1 The Simulating Method $\ldots \ldots \ldots$. . . . . . . 50

6.4 .2 Results . . . . . . . . . . . . . . . . . 52

7 Summary and Future Work $\quad 57$

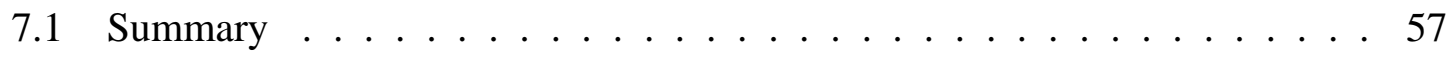

7.2 Future Work . . . . . . . . . . . . . . . . . 57 


\section{List of Figures}

3-1 A simple illustrative example . . . . . . . . . . . . . . . . 22

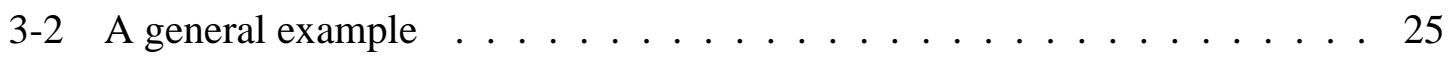

4-1 How to recognize congested links: An example . . . . . . . . . . . . 29

$4-2 \quad N_{i}, \frac{\sum_{j \in i} \lambda_{j}}{C_{i}} \sim \alpha \ldots \ldots \ldots \ldots \ldots \ldots \ldots \ldots \ldots \ldots \ldots \ldots \ldots$

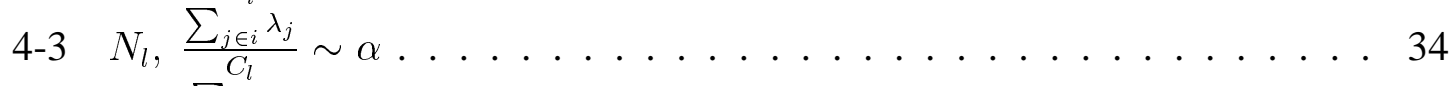

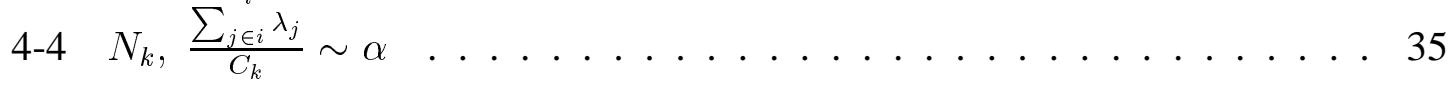

5-1 A Redundant Constrain: $\left(\lambda_{1}+\lambda_{2} \leq C_{3}\right) \ldots \ldots \ldots \ldots$. . . . . 40

6-1 Simulation Results of a Low Speed Net . . . . . . . . . . . . . . . . 49

6-2 Simulation Results of a High Speed Net . . . . . . . . . . . . . . 49

6-3 Simulation Results of a Low Speed Net: $\Delta t=0.30 \mathrm{~s} \ldots \ldots \ldots$

6-4 Simulation Results of a High Speed Net: $\Delta t=0.25 \mathrm{~s} \ldots \ldots \ldots \ldots$

6-5 Simulation Results of a Low Speed Net: $\Delta t=0.50 \mathrm{~s} \ldots \ldots \ldots$

6-6 Simulation Results of a High Speed Net: $\Delta t=0.50 \mathrm{~s} \ldots \ldots \ldots$ 


\section{List of Tables}

4.1 Each flow's static round trip delay in Figure 4-1 . . . . . . . . . . . . . . . 29

5.1 Results Comparison: Queue Lengths . . . . . . . . . . . . . . . . 43

5.2 Results Comparison: Average rates of flows . . . . . . . . . . . . 43 


\section{Chapter 1}

\section{Introduction}

\subsection{Motivation}

The Internet has been growing rapidly in recent years, which results in problems related to routing, flow control, administration etc. Simulation and analysis are the two commonly used methods to study the characteristics of networks. However, when networks become very large, neither method is easy to implement. For example, the traditional packet by packet level event driven simulation is slow when simulating a high speed network. The fixed granularity of the event driven simulation is the bottleneck of its speed. Unless we change the abstraction level, it is hard to improve its performance. Analytic method often uses classic queuing and network stochastic models which are restricted to problems that can be approximated as Markov chains [1]. As networks are using complicated protocols such as TCP/IP to implement flow control, they can't be simply modeled by Markov chain assumption. Thus, we lack a satisfying model to study the behavior of window based flow control packet-switched networks.

The goal of this thesis is to develop a simple and applicable model for window based flow control packet-switched networks under some reasonable assumptions. The model is expected to ignore the packet-level behavior of networks. Instead, it gives a higher level description, such as the average flow rate and the average queue length at each outgoing link. Incorporating this model into simulation methods will hopefully provide a practical way to study the behavior of large scale networks. 


\subsection{Organization of this Thesis}

Chapter 2 is a review of the related work. Chapter 3 introduces the model. It includes basic assumptions the model depends on and it also describes the meaning of every formula in the model. Chapter 4 presents an algorithm for applying the model. Chapter 5 discusses the implementation details. Chapter 6 shows how to apply this model to simulate TCP with RED. The last chapter is a short summary of the thesis and an assessment of future work. 


\section{Chapter 2}

\section{Related Work}

\subsection{Simulation Methods}

As networks become larger and more complex, more and more researchers are using simulation to conduct the study of networks. Two effective ways are used for network simulation. One is the discrete event driven simulation. The other is the discrete time driven simulation. Since in most networks, data are chopped into discrete packets, discrete event driven simulation is straightforward and it often can grasp the nature of networks.

The current academic-wide popular network simulator, $n s$ [3], developed by the Network Research Group at Lawrence Berkeley National Laboratory(LBNL), is part of the Virtual InterNetwork Testbed(VINT) project [2]. The goal of VINT is to build a network simulator which will facilitate the study of scale and protocol interaction of modern network protocols. $n s$ is a discrete event driven simulator which can simulate a wide range of protocols, such as TCP and other routing, multicast protocols. In this thesis, $n s$ is used to test the model.

The representation of an event in $n s$ is the state of a packet. Typical events could be the arrival or departure of a packet from a queue. Thus, detailed information of each packet's behavior can be obtained via simulation. $n s$ has a global scheduler which manages an event queue. This queue is arranged in the time sequence that the events should happen. The simulation proceeds by simulating each event in the order they are stored in the queue. As one event takes place, it can trigger other events. These events are also inserted into the 
queue based on the time they are to happen. For the packet level event driven simulation, the simulator has to simulate each packet's behavior even if we are not interested in such a fine abstraction level.

When the network's size is moderate, $n s$ is an ideal tool to study network protocols. The detailed information about each packet's trace, such as when the packet arrives at a specific router, or when and where the packet is dropped, can be precisely recorded. However, discrete event driven simulation is slow when the speed of networks is high. First, the packet level granularity is fixed in spite of the size of the network. Second, in order to update the effects caused by each event, the event order must be kept. A global event list has to be maintained, which results in an essentially sequential process. These two phenomena can not be avoided in the discrete event driven simulation.

A discrete time driven simulation updates the state of each simulated object at each delta time interval. Choosing a proper time interval is always a difficult problem. If the time interval is too large, the simulation will fail to detect events which should have occurred during the time interval. If such events are significant, the simulation's error bar will be high. On the contrary, as the simulator has to update the state of each simulated object at each delta interval, if the delta interval is too small, the simulator has to do the updating very frequently. If we have a large network which contains a lot of objects, since not every object changes its state so frequently, the simulation is very ineffective. Especially when we are interested in a long time period simulation, it may also take a long time to do so many updatings.

Another proposal is to use fluid model based time-driven simulation to simulate high speed networks [7]. This model simulates the network traffic as fluid. It is proved that the discretization error can be bounded by a constant proportional to the discretization time interval. This model can quickly locate the time and place of congestion. Besides, by the nature of time driven simulation, parallelism can be exploited to speed up the simulation. However, under what circumstance the network traffic can be modeled as fluid still needs further study. In the window flow control network, the rates of flows are unknowns. The fluid model needs the arrival rates of flows as input. We do not know whether the fluid model is adequate enough to model the window based flow control networks. 


\subsection{Queuing Theories}

Also, there are a lot of well developed queuing and stochastic network theories related to network flow and congestion control. When the network is large, those analytic models tend to contain some impractical assumptions such as Markov chain. Thus, it is not convenient to apply such queuing theories to window flow control networks.

\subsection{Summary}

In a word, for large scale and high speed window flow control networks, there is no satisfying model. The task of this thesis is to construct an analytic model for such networks. We will discuss it in the next chapter. 


\section{Chapter 3}

\section{Model Construction}

This chapter describes the model for window based flow control networks. We first give a brief overview of the end-to-end window based flow control mechanism that is used by TCP. Next, we present an abstraction of the real TCP. Our model is applicable under some assumptions. Since the network's steady state is the point of interest, omissions of implementation details of TCP will not hurt the model's praticability.

\subsection{End-to-end Window Based Flow Control}

A flow of data between a sender A and a receiver B is said to be end-to-end window flow controlled if there is an upper bound on the data units that have been sent by A but are not known by A to have been received by $\mathrm{B}$ [1]. The upper bound is called window size. In TCP, the upper bound is used both to keep the sender from overflowing the receiver's buffer and to keep the sender from overflowing the buffers inside the network. The sender keeps two window sizes for the two purposes. The congestion window reflects the congestion condition in the network. When the network is under heavy congestion, by reducing the congestion window size, the sender holds the data units from entering the network. The advertised window tells the sender the available buffer size in the receiver's side. The sender's real window size should never exceed either of them. TCP uses ACKs from the receiver to inform the sender of the correct receipt of data packets. When there is a full window size of packets on fly, the sender can not advance its window until it receives an 
ACK from the receiver.

Window based flow control can not guarantee a minimum sending rate nor a minimum packet delay. When the network is suffering congestion, packets are queued up inside the network. It will take a packet a long time to go through the network. So does the ACK. If the window size is fixed, the longer the delay is, the lower the throughput is. And if there are many senders competing for the limited links' capacities, each sender can only get a small transmitting rate. Thus packets are blocked in output links. The packet delay is increased with the increase of waiting queues.

\subsection{Basic Assumptions}

Though window flow control does not guarantee rate and delay, in some circumstances, we are still interested in knowing how much the average rates window-controlled flows could achieve and how large the queue size could be at each outgoing link if each flow's window size is known and fixed. Doing packet by packet level simulation is a way to find out those parameters. However, simulation is usually time consuming. We aim at a simple model which can provide us the steady state information fast and fairly accurately.

As we are interested only in the steady state, we want to give some assumptions to clarify the situation we are studying.

- Network configurations: assume we know the topology of the network, each link's capacity and propagation delay.

- Routing and Queuing Policy:

1. Assume fix route routing. All packets belonging to the same sender and receiver travel through the same path. The routing table of each router is known.

2. Assume routers keep a distinct output queue for each outgoing link. The processing delay of each packet at the router is negligible compared to the propagation delay, queuing delay and transmission delay.

3. The output buffer is infinite. We do not consider buffer overflow for now. 
4. All data packets are of the same priority class. They are all arranged into a single queue and first come, first transmitted.

- Flows:

1. All flows have fixed window sizes, and the network is in a steady state, e.g., each flow has a full window size of packets on fly and the packets are spaced evenly.

2. Senders always have data to send. In the period of our study, no flows stop sending data and no new flows start sending.

3. There is only one-way traffic flow. When a receiver receives a data packet, it sends an ACK back immediately. ACKs travel through the same paths as those of their acked data packets ${ }^{1}$. Since ACKs are tiny packets, we assume ACKs are never backlogged at any link's output queue.

- Packets: All packets are of the same length. In this thesis, we assume the packet length is 1 Kbyte.

In the next section, we discuss our model based on the above assumptions.

\subsection{The Model}

We start from a simple example to get some intuition. Figure 3-1 shows the example. There is only one sender which is sending with a window size $W$. By the network's steady state assumption, the flow has $\mathrm{W}$ packets on fly. They are either inside the pipe, transmitted by $\operatorname{sr} c_{1}$ or $r_{1}$ or be queued at $r_{1}$. Since the flow is sending packets evenly spaced, the number of packets in the pipe is roughly $\left(\frac{t_{1}}{\frac{1}{\lambda}}+\frac{t_{2}}{\frac{1}{\lambda}}\right)(\lambda$ denotes the flow's rate, e.g., the number of packets the flow sends per unit time). $t_{1}, t_{2}$ are link 1 and 2 's propagation delay. And the number of returning ACKs is also $\frac{t_{1}}{\frac{1}{\lambda}}+\frac{t_{2}}{\frac{1}{\lambda}}$. If link 2 is the bottleneck link, the other packets in

\footnotetext{
${ }^{1}$ This assumption is not necessary. It only means to simplify programming. If ACKs travel through symmetric paths as those of data packets, only the forwarding paths of data packets need to be specified. The model is also applicable when ACKs travel through asymmetric paths. The only difference is we have to specify both data and ACKs' paths in order to calculate the round trip delay.
} 


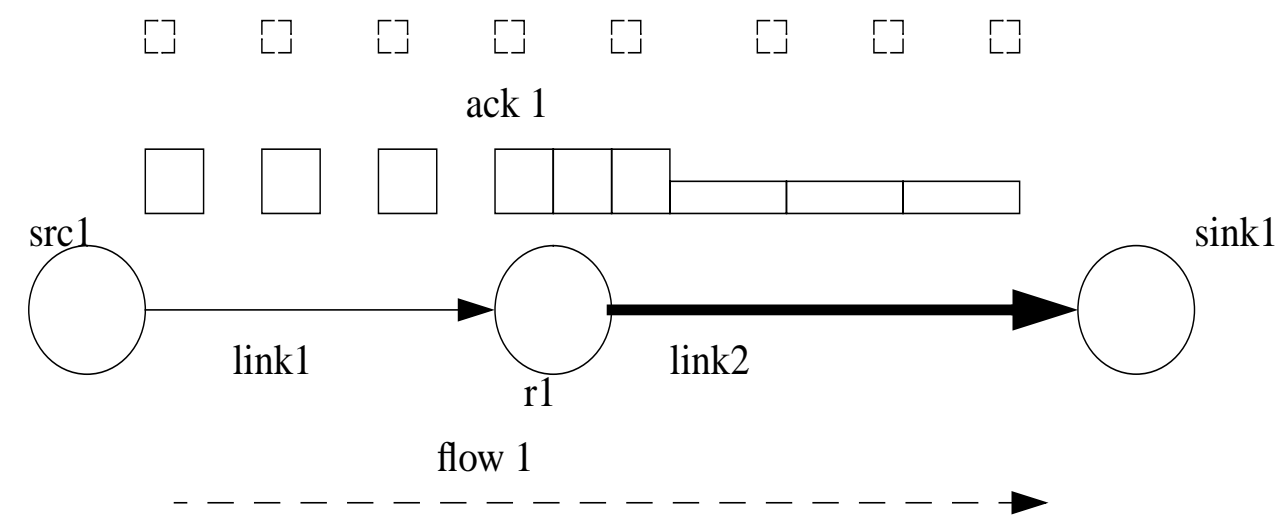

Figure 3-1: A simple illustrative example

the window will be queued or transmitted at $r_{1}$. If the flow does not change its window size, the state will persist. The same number of packets and ACKs are distributed along the pipe and the queue. Since we know link 2's capacity and we figure out it is the flow's bottleneck link, we conclude the flow's steady state sending rate is equal to the link's capacity. Then we know immediately how many packets are in the pipe and how many packets are at $r_{1}$ without packet by packet level simulation.

In general, if we know which links are bottleneck links, we can always calculate the output queue length by similar computation ${ }^{2}$. It gives us an easy way to obtain the steady state information.

Before explaining our approach for more general cases, we define some symbols.

- $W_{j}$ : window size of flow $j$

- $P_{j}$ : the static round trip delay of flow $j$, which is the sum of the link's propagation delay and transmission delay along the path of flow $j$. Because packets are of the same size, one link's transmission delay for a packet is a constant. We can view the transmission delay as an extension of the pipe's length.

- $\lambda_{j}$ : the steady state rate of flow $j$.

- $p_{j}$ : the path of flow $j$, which contains links flow $j$ crosses.

\footnotetext{
${ }^{2}$ In this thesis, a bottleneck link refers to a link which is fully loaded. Sometimes we call it a congested link because such a link usually has a backlogged packet queue. However, it is possible in some case, a link is fully utilized but not congested. We do not distinguish them strictly.
} 
- $N_{i}$ : number of backlogged packets at outgoing link $i$. If the link $i$ is a congested link, $N_{i}>0$. Otherwise, $N_{i}=0$.

- $n_{j i}$ : number of backlogged packets from flow $j$ at link $i$. If the link $i$ is a congested link, $n_{j i}>0$. Otherwise, $n_{j i}=0$.

- $C_{i}$ : capacity of link $i$.

According to the assumptions, we have:

For a flow $j$,

$$
W_{j}=\frac{P_{j}}{\frac{1}{\lambda_{j}}}+\sum_{i \in p_{j}} n_{j i}
$$

For a link $i$,

$$
N_{i}=\sum_{j \in i} n_{j i}
$$

Nevertheless, in a general case, we usually do not know each flow's steady state's sending rate. In fact, it is an unknown we want to compute. Thus, from Equation 3.1 and 3.2, we can not easily get $n_{j i}$ nor $N_{i}$. However, $\lambda_{j}$ and $n_{j i}$ are related. They satisfy a set of physical constraints. The extra constraints provide a way to find out rates of flows and queue lengths at congested links.

As we know, if link $i$ is not congested,

$$
\begin{aligned}
n_{j i} & =0, \forall j \in i \\
N_{i} & =0
\end{aligned}
$$

If link $i$ is congested, the link must be sending at its full capacity. So,

$$
C_{i}=\sum_{j \in i} \lambda_{j}
$$

If link $i$ is not congested,

$$
C_{i} \geq \sum_{j \in i} \lambda_{j}
$$

In the steady state, if a link $i$ is congested, each flow $j$ across link $i$ has a constant number of packets queued at it. The more backlogged packets one flow has at link $i$ 's output 
queue, the more chance it has to get a packet transmitted; hence the more throughput the flow could achieve. This observation follows because all packets are of the same length. More clearly, we have:

$$
\frac{n_{j i}}{N_{i}}=\frac{\lambda_{j}}{C_{i}}, \forall N_{i} \neq 0
$$

Notice we can use Equation 3.7 to substitute $n_{j i}$ in Equation 3.1, we finally get:

$$
W_{j}=\lambda_{j}\left(\sum_{i \in p_{j}} \frac{N_{i}}{C_{i}}+P_{j}\right)
$$

which is exactly the result from the Little's Theorem. We illustrated it in an intuitive way.

Equation 3.5 and 3.8 are fully constrained. The total number of unknowns are the sum of the number of flows and the number of congested links. Corresponding to each flow, we have an Equation 3.8. And for each congested link, we have an Equation 3.5. The number of unknowns and the number of equations are matched.

Thus, if given a network configuration and each flow's window size, the steady state is deterministic, we claim in steady state, the rate of each flow and the queue length at each outgoing link must satisfy the above equation set and all the other constraints such as Inequality 3.6 and Equation 3.4. On the other hand, if a solution satisfies all the equal and unequal constraints, it must be the steady state parameters.

For example, in Figure 3-2, the set of constraints obtained from modeling is:

$$
\left\{\begin{array}{l}
W_{1}=\lambda_{1}\left(\sum_{i=1,2} \frac{N_{i}}{C_{i}}+P_{1}\right) \\
W_{2}=\lambda_{2}\left(\sum_{i=1,3} \frac{N_{i}}{C_{i}}+P_{2}\right) \\
W_{3}=\lambda_{3}\left(\sum_{i=3,4} \frac{N_{i}}{C_{i}}+P_{3}\right) \\
C_{i}=\sum_{j \in i} \lambda_{j}, \forall N_{i}>0, \text { e.g., link } i \text { is congested. } \\
C_{i} \geq \sum_{j \in i} \lambda_{j}, \forall N_{i}=0, \text { e.g., link } i \text { is not congested. }
\end{array}\right.
$$

However, we still face a difficult problem. We do not know how to find out a solution 


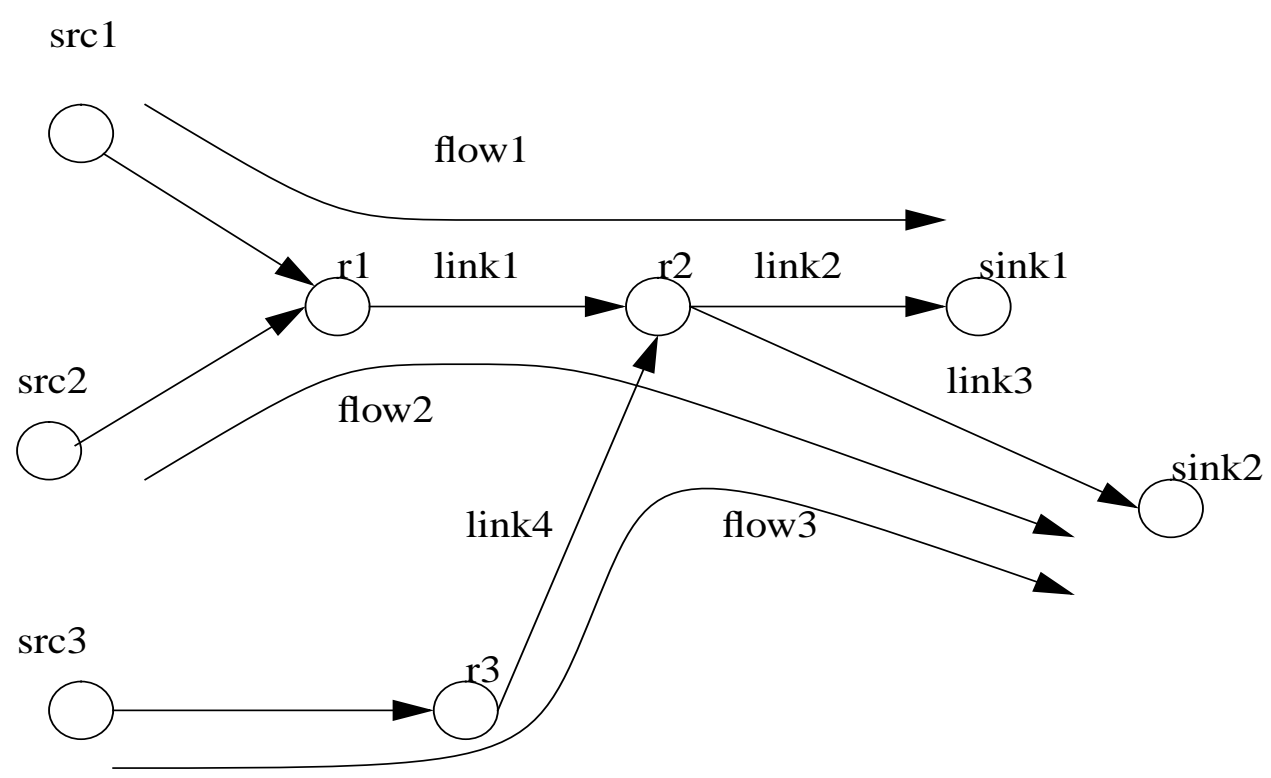

Figure 3-2: A general example

for the model. The model consists of multidimensional non-linear equations 3.8 subject to linear constraints $3.5,3.6,3.4$. If we can identify the congested links, the problem will be reduced to finding a root for a set of non-linear equations. It is much easier. We will discuss the algorithm of identifying the congested links in Chapter 4. 


\section{Chapter 4}

\section{Algorithm}

In Chapter 3, we introduced the model. The model mainly consists of two parts. The first part is:

$$
W_{j}=\lambda_{j}\left(\sum_{i \in p_{j}} \frac{N_{i}}{C_{i}}+P_{j}\right)
$$

In the second factor of the left hand side, the first term $\sum_{i \in p_{j}} \frac{N_{i}}{C_{i}}$ is the total queuing delay. The second term is the static round trip delay. The sum of the two is the average round trip delay of this flow. Thus this part of the model states the fact that in the steady state, a flow sends a window size of packets every round trip time. The flow's round trip delay is determined by its own path and the congestion condition of the network, which depends on the total load of the network.

The second part is:

$$
\begin{aligned}
& \left\{\begin{array}{l}
N_{i}=0 \\
C_{i} \geq \sum_{j \in i} \lambda_{j}, \text { if link } i \text { is not congested }
\end{array}\right. \\
& \left\{\begin{array}{l}
N_{i}>0 \\
C_{i}=\sum_{j \in i} \lambda_{j}, \text { if link } i \text { is congested }
\end{array}\right.
\end{aligned}
$$

The second part states the feasible constraints. A link can not send faster than its capacity. If the incoming flows require more transmission rate than what the link can handle, 
their packets are backlogged. As flows are window controlled, they can not put more than a window size of packets into the network. Thus flows can not send faster than the returning rates of ACKs. As a result they will not further overflow the link. The number of backlogged packets are stable and the link will send at its full speed because every packet it transmits from the queue will trigger a new incoming packet.

To obtain the steady state parameters from this model, we need to find a satisfactory solution for the formulas. It is not an easy task. We discuss it in the following sections.

\subsection{The Difficult Point}

As mentioned in Section 3.1, window flow control does not guarantee a minimum sending rate nor a minimum delay. This means there is no simple linear relation between window size and the flow's sending rate or round trip delay. We can also see it from Equation 4.1. Without changing the window size, the flow's rate could become very low while the round trip delay becomes very huge. However, as the total number of packets inside the network is fixed, which is the sum of all flows' window sizes, the queuing delay is determined by both this number and the network configuration. Therefore, each flow's queuing delay can not be arbitrary. If we can figure out how packets are distributed along the pipe and backlogged at each output queue, we can calculate the queuing delay and further more, the steady state rates. We know that packets are backlogged if and only if the outgoing pipe is full, which is said by the second part of the model. Combining the two parts, we can find a set of steady state parameters. The difficult point is how to locate fully utilized links. The rates of flows are shaped by those links, which is the same phenomenon as TCP's self-clocking mechanism [4].

There is a simple and attractive idea. For each flow $j$, the maximum rate it could reach

is $\frac{W_{j}}{P_{j}}$. This happens when there is no congested link along its path. This rate can be viewed as the required rate of this flow. For each link, if the sum of required rates from all incoming flows exceed its capacity, we could conclude the link is the congested link. However, this is not true. Since the real rates will be reduced by congestion, a flow might achieve a much lower sending rate than the required one. Thus, some link may seem to be 


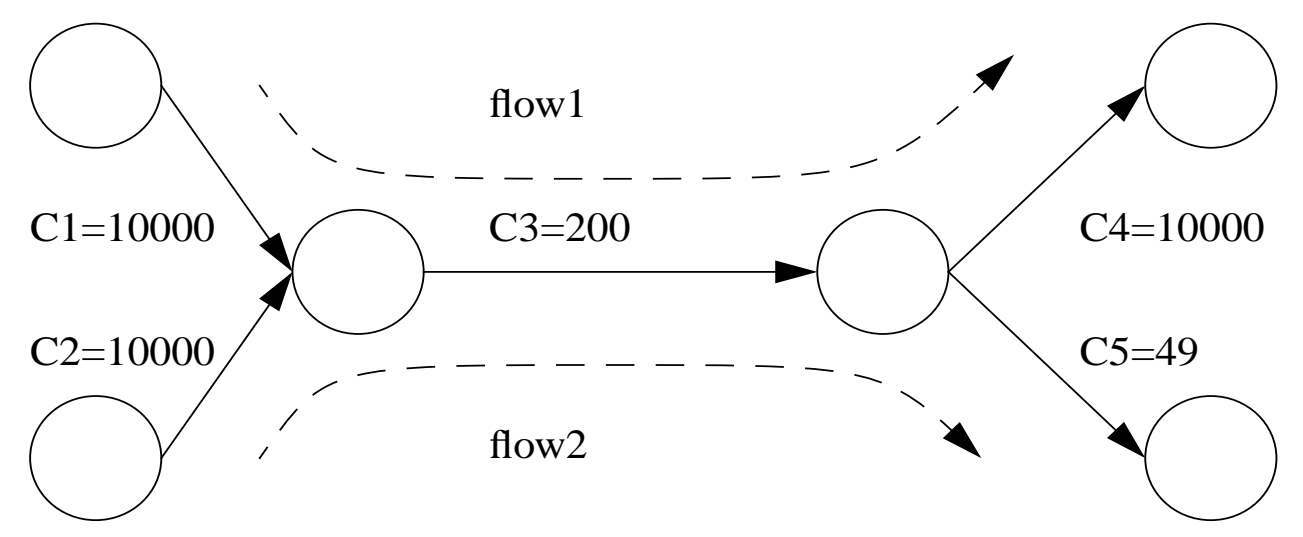

Figure 4-1: How to recognize congested links: An example

\begin{tabular}{|c|c|c|}
\hline flow index & window size (packets) & static rtt (ms) \\
\hline 1 & 41 & 165 \\
\hline 2 & 5 & 45.5 \\
\hline
\end{tabular}

Table 4.1: Each flow's static round trip delay in Figure 4-1

congested but in fact it will not be. Even the seemingly 'most congested link' may turn out to be non-congested at all. We will give one example to demonstrate this point.

In the example, the network's topology, each flow's route and each link's capacity are shown in Figure 4-1. Each flow's window size and static round trip delay(the sum of link's propagation delay and transmission delay along a flow's path) are shown in Table 4.1

The required rate of flow 1 is $\frac{41}{0.165} \doteq 248.5$ packets/sec. The required rate of flow 2 is $\frac{5}{0.0455} \doteq 109.9$ packets/sec. If we define the most congested link as the one which has the maximum $\frac{\left(\sum_{j \in i} \lambda_{j}-C_{i}\right)}{C_{i}}$, link 5 is the most congested one $((109.9-49) / 49 \doteq 1.24>$ $(248.5+109.9-200) / 200 \doteq 0.79)$. However, in the steady state, the real congested link is link 3 . The queue length at link 3 is roughly 17.5 packets. So, flow 1 's rate is reduced to $\frac{41}{17.5 / 200+0.165} \doteq 162.5$ packets/sec. Flow 2's rate is $\frac{5}{17.5 / 200+0.0455} \doteq 37,5$. Link 5 is not congested.

This example intends to show the difficulty of locating congested links. To make the model work, we have to develop an algorithm which can solve the problem. 


\subsection{How to Find a Solution for the Model}

\subsubsection{Ideas}

When the network has reached a steady state, if we 'remove' all the backlogged packets and reduce each flow's window size correspondingly, the network will still stay in the steady state and each flow will remain the same sending rate. This is the threshold for the network to go from non-congestion to congestion. At this point, though some links are sending at their full speed, they do not have backlogged packets. If we can catch this threshold, we can easily get each flow's steady state sending rate since there is no queuing delay yet and the round trip delay of each flow is equal to the static round trip delay. Starting from the threshold, if we inject packets into the network by increasing flows' window sizes to their original ones, packets will be queued up only at those links which are sending at the full speed at the threshold point. That is, the congested links in the steady state are the links that are sending at the full speed at the threshold point. This idea provides us some insight. We can add a light load to the network at the beginning. Next, we increase the load gradually and see whether there are some links that are reaching their capacities. We keep a record of those links until we increase the load to the given value. We use our record and the equation set of the model to get the steady state parameters. The details of this algorithm are discussed in the next section.

\subsubsection{Details}

We multiply each flow's window size by a scaling factor $\alpha(\alpha \in[0,1])$ and substitute $W_{j}$ in Equation 4.1 with $\alpha W_{j}$. Let $\operatorname{Cong}(\alpha)$ denotes the set of congested links for the value $\alpha$. Obviously, $\operatorname{Cong}(0)=\Phi$. Let $E(\alpha)$ be a set of equations chosen from the constraints. Let $n$ be the number of flows. At each value of $\alpha, E(\alpha)$ has $n$ equations of the form:

$$
\alpha W_{j}=\lambda_{j}\left(\sum_{i \in p_{j} \text { and } i \in \operatorname{Cong}(\alpha)} \frac{N_{i}}{C_{i}}+P_{j}\right)
$$

Other equations in $E(\alpha)$ are of the form: 


$$
\sum_{j \in i} \lambda_{j}=C_{i}, \forall i \in \operatorname{Cong}(\alpha)
$$

The algorithm works as follows.

- Step 1: increase $\alpha$ to $\alpha+\delta$.

- Step 2: solve $E(\alpha+\delta)$ assuming $\operatorname{Cong}(\alpha+\delta)=\operatorname{Cong}(\alpha)$.

- Step 3: check the feasible constraints and update $\operatorname{Cong}(\alpha)$ to $\operatorname{Cong}(\alpha+\delta)$ based on the following rules. For link $i \ni^{1} \operatorname{Cong}(\alpha)$, if $\left(\sum_{j \in i} \lambda_{j}-C_{j}\right) \geq 0$, update Cong by adding $i$ into it. For link $i \in C o n g$, if $N_{i} \leq 0$, delete it from Cong. If neither happens, e.g., no link is overloaded and no queue size is below zero, Cong remains the same.

- Step 4: update $E$ based on the current $C o n g^{2}$.

- Step 5: if $\alpha=1$, solve $E$ and stop. Otherwise, go to Step 1.

By induction, we prove that when $\alpha=1, \operatorname{Cong}(1)$ is the set of congested link when every flow $j$ 's window size is $W_{j}$. Therefore, the feasible solution of $E(1)$ is the solution of the original equations and inequalities of the model.

Initially, because $\operatorname{Cong}(0)=\Phi . E(0)$ only contains

$$
0 \times W_{j}=\lambda_{j} P_{j}, j=1,2, \ldots, n
$$

The solution of $E(0)$ is $\lambda_{j}=0$ for any flow $\mathrm{j}$. Because no link is congested, $\operatorname{Cong}(0)=$ $\Phi$ is the right congested link set.

Suppose when $\alpha$ reaches some value, $\operatorname{Cong}(\alpha)$ is the right set of congested links. We prove when $\alpha$ is increased by a tiny value $\delta$, our algorithm will correctly update $\operatorname{Cong}(\alpha)$ and as a result the solution of $E$ will give the steady state parameters for the situation each flow $j$ 's window size is reduced to the new value of $\alpha W_{j}$.

\footnotetext{
${ }^{1} \ni$ means 'not a member of'.

${ }^{2}$ Details are discussed in Chapter 5
} 
When $\alpha$ is increased by a tiny value, inside the network, three possible situations would occur.

First, the old congested links remain congested. The newly added load are either queued at those old congested links or make other non-full pipes more crowded but not full, which means, no new congested link occurs. In this case, the set of congested links does not change. If we use the old $E(\alpha)$ set to get a new solution for $\alpha+\delta$, there will be no overflow for non-congested links and the old queue sizes are still positive since the old links are still congested. We do not change Cong in this case. And Cong is still the right set of congested links for the new value of $\alpha$.

Second, the new load makes some pipe full. Correspondingly, some new congested link occurs. As the old Cong does not contain the new congested link, the solution of $E$ based on the old Cong will not satisfy $\left(\sum_{j \in i} \lambda_{j}-C_{j}\right) \leq 0$ for the new congested link. So, if we find out some constraint is violated, we can conclude the pipe must be filled up by the new $\alpha$ and we add the link into Cong.

Third, the new load makes some congested link non-congested. It sounds impossible at first. How could it happen while we add more load and a congested link become noncongested? The answer is that when a flow puts more packets into a congested link, all flows sharing the same link will suffer more queuing delay and as a result, their sending rates will be slowed down. Consequently, if those flows go through other links after transmitted by this link, they add less load to those links. Then it is possible for some former congested link to change to non-congested. When we solve $E$ in the new value of $\alpha$ and the old set of congested links, we tend to get a very small queue length or a negative queue length. Because the link is in the congested link set, which means we assume its pipe is full, if we still get a positive queue length, that is, the pipe can not accommodate all packets. So the link should still be congested at that point. The negative queue length implies the link should not be congested for the new $\alpha$. We delete it from Cong.

As we update Cong correctly in according to all possible situations, Cong is still the right set of congested links.

Then we induce that $\operatorname{Cong}(1)$ is the right set of congested links when $\alpha=1$ and finish the proof. 


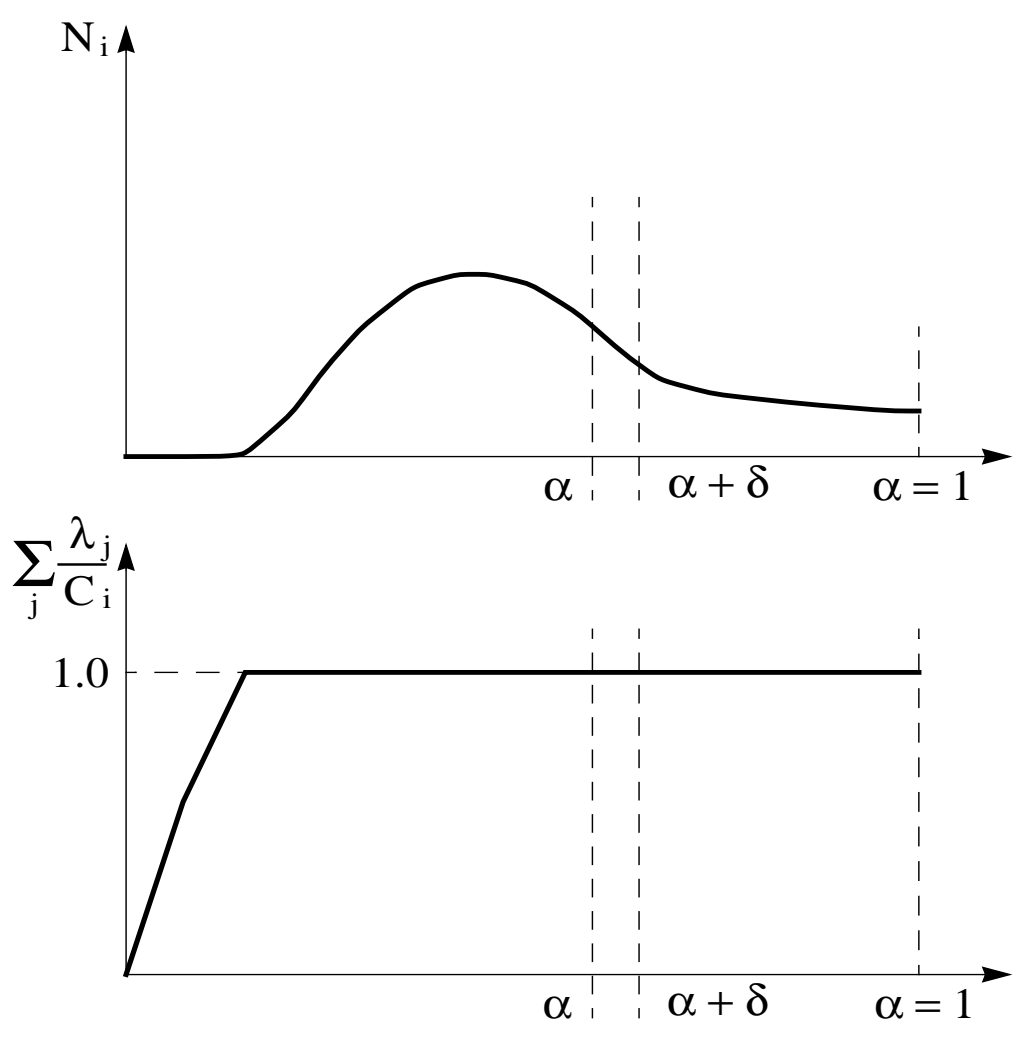

Figure 4-2: $N_{i}, \frac{\sum_{j \in i} \lambda_{j}}{C_{i}} \sim \alpha$

\subsubsection{Another Perspective of the Algorithm}

The algorithm can be viewed from another perspective. Each flow's rate and each link's queue length are functions of $\alpha$. They change dependently. As shown in Figure 4-2, 4-3 and 4-4, when the sum of flows' rates at a link is leveled out by the link's capacity, the link's queue starts increasing from zero. When the queue is drained out and becomes zero, the link is underloaded after that. The functions are non-smooth however. They are described by different formulas in different intervals of $\alpha$. This is the reason why the equation set $E(\alpha)$ is also a function of $\alpha$. But the changing points of a link's queue length and its total load are the same. At the changing points, both the left side's constraints and the right side's ones are valid. For example, if at a value of $\alpha_{1}$, a link $i$ starts to be congested, we can either insert $i$ into $\operatorname{Cong}\left(\alpha_{1}\right)$ or not. Because $N_{i}=0$ and $\sum_{j \in i} \lambda_{j}=C_{i}$ are both true when $\alpha=\alpha_{1}$, if we insert $i$ into $\operatorname{Cong}\left(\alpha_{1}\right)$, and add unknown $N_{i}$ and Equation 4.3 of $C_{i}$ into $E\left(\alpha_{1}\right)$, the right solution has to satisfy $N_{i}=0$; if $\operatorname{Cong}\left(\alpha_{1}\right)$ has not contained link $i$ yet, since we force $N_{i}=0$, the right solution of $\operatorname{Cong}\left(\alpha_{1}\right)$ must also satisfy $\sum_{j \in i} \lambda_{j}=C_{i}$, 

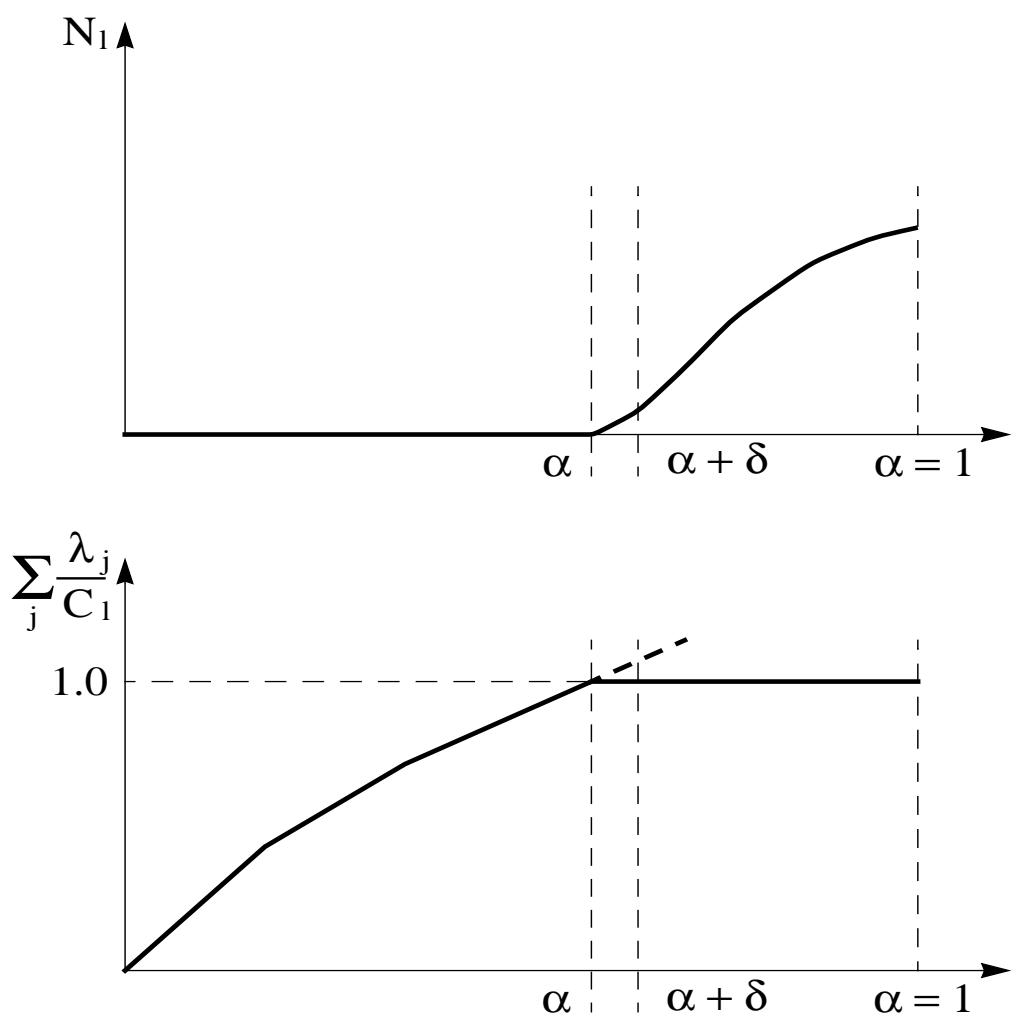

Figure 4-3: $N_{l}, \frac{\sum_{j \in i} \lambda_{j}}{C_{l}} \sim \alpha$

otherwise, $N_{i}=0$ and $\sum_{j \in i} \lambda_{j}=C_{i}$ can not be both true, which is contradictory to the feasible constraints. Similarly, if at some point, link $i$ begins to be underloaded, whether we keep it in the $\operatorname{Cong}(\alpha)$ or delete it will both satisfy the constraints.

Though the rates of flows and the queue lengths of links are non-smooth functions of $\alpha$, for each value of $\alpha, E(\alpha)$ consists of smooth continuous functions of $\alpha$. By the continuousness of a smooth function, if for some value of $\alpha, \sum_{j \in i} \lambda_{j}=C_{i}$ is true, within a small neighborhood of $\alpha$, such as $[\alpha-\delta, \alpha+\delta], C_{i}-\epsilon<\sum_{j \in i} \lambda_{j}<C_{i}+\epsilon$ is also true. Figure 4-3 gives an example. We assume at point $\alpha$, link $l$ is not congested. The induction assumption says it is correct. For this value of $\alpha$, the set of constraints in $E$ is a set of continuous smooth functions. If at the two end points of the interval $[\alpha, \alpha+\delta]$, $\sum_{j \in i} \lambda_{j}$ changes from less than $C_{l}$ to over $C_{l}$, we deduce there must be a $\alpha^{\prime} \in[\alpha, \alpha+\delta]$, which satisfies $\sum_{j \in l} \lambda_{j}=C_{l}$. As discussed above, this indicates the changing point of the congestion condition of link $l$. By adding link $l$ into $\operatorname{Cong}(\alpha+\delta)$, we correctly identify the new congested link. The same analysis is applicable when a link is changed from 

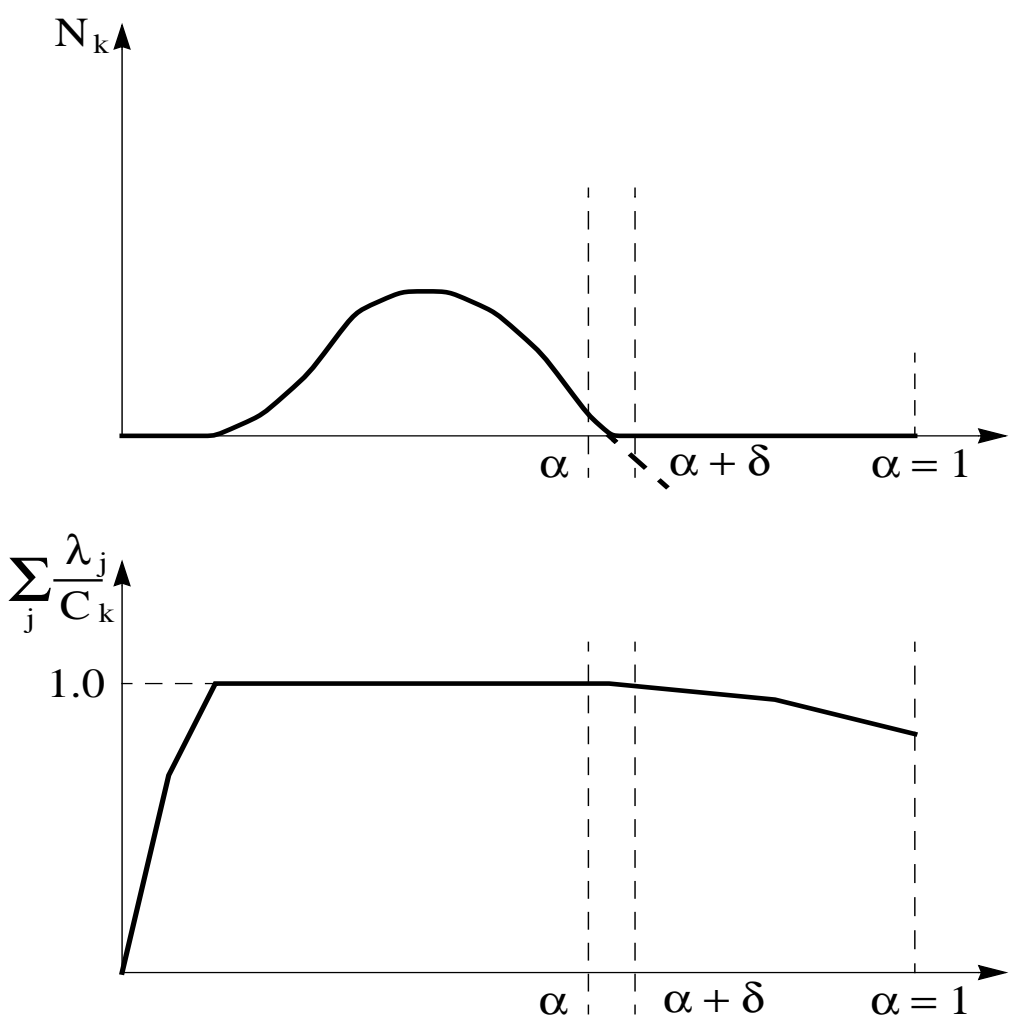

Figure 4-4: $N_{k}, \frac{\sum_{j \in i} \lambda_{j}}{C_{k}} \sim \alpha$

congested to non-congested. As the solution of $E$ guarantees the load of a congested link is its capacity, the change of queue length is used as the indication instead. When a congested link's queue length goes to negative, it implies the link is going to be non-congested under the new value of $\alpha$ (See in Figure 4-4). In both cases, the solutions of $E(\alpha)$ assuming $\operatorname{Cong}(\alpha)=\operatorname{Cong}(\alpha+\delta)$ are the extrapolation of the set of constraint functions in $E(\alpha)$ (Figure 4-3 and 4-4).

\subsection{Short Discussion About the Implementation}

At each iteration, the algorithm involves two major operations. One is to solve the equation set $E$ and the other is updating Cong. The number of iteration is $\delta^{-1}$. To make the algorithm efficient, we need to reduce the number of iterations. As the network of windowbased flow control is in fact a non-linear system, in general, it is always hard to obtain state parameters. In the next Chapter, we will discuss several implementation issues. 


\section{Chapter 5}

\section{Implementation Issues and Testing Results}

This chapter addresses detailed implementation issues. There are two major concerns about the implementation. To test the correctness and efficiency of the model, we run $n s$ on same testing cases and compare the results from $n s$ with those of the model. The comparison shows the model correctly grasps the steady state characteristics of window based flow control networks. With the current implementation, the model's performance is fairly good though there is still some potential to improve it.

\subsection{How to Reduce the Number of Iterations}

The first concern is the number of iterations. Our algorithm relies on the continuousness of constraint functions in $E$. If we choose a large increment of $\alpha$ to reduce the number of iterations, we may miss some changing points, e.g., some links' congestion conditions may have changed more than once. Then the results we compute from the old congestion set Cong may not indicate the right modification. Thus, we are not sure whether the updating based on those results are correct. Our induction of the correctness of the algorithm does not work. On the other hand, if we choose a small increment, the number of iterations is huge and the model is not efficient. Besides, if the network's congestion state does not change very rapidly, there is no need to update the set of congested links so frequently. 
We employ the idea of a binary root finding algorithm to solve the dilemma. We keep two variables. One is left and the other is right. We first choose a large step of increment. We let left equal to the $\alpha$ of last iteration and right equal to 1. We assume the old set of congested links are still valid and solve the equations in $E$ for the the value of right. We check all feasible constraints of the model. If they are all satisfied, we believe we have chosen the right congested links and get the right solution and we stop the iteration. If some of the constraints are violated and we can not tell how to modify the set of congested links, we try a new value of $\alpha, \alpha=\frac{\text { left }+ \text { right }}{2}$. If all constraints are satisfied under this new value of $\alpha$, we increase left to the current $\alpha$ and set $\alpha$ to the middle of the new left and right and try again. If some constraints are violated and we are not sure how to update the Cong, we decrease right to current $\alpha$ and set $\alpha$ to the middle of left and the new right and try again. If some constraints are violated and we know how to update the congested links, we update the congested links and start the next iteration with the new $\alpha$ and the congested link set Cong.

We use two criteria to decide whether it is suitable to change the set of congested links based on the solution obtained from the old set of congested links. The first one is strict. The second one is heuristic. As we know, the feasible constraints we check for after we obtain a solution from a old Cong are :

$$
\begin{aligned}
& N_{i} \geq 0, \forall i \in \text { Cong } \\
& C_{i} \geq \sum_{j \in i} \lambda_{j}, \forall i \ni \text { Cong }
\end{aligned}
$$

If some constraints are violated but within a small range, such as $\operatorname{Min}\left(N_{i}\right) \geq-\epsilon$ and $\operatorname{Max}\left(\frac{\sum_{j \in i} \lambda_{j}-C i}{C_{i}}\right) \leq \epsilon$, we believe the old results are valid to direct the modifications. We update the congested links as we stated in Section 4.2.2. This is based on the continuous assumption of constraint functions.

The second criterion for us to update the congested links is when there is only one constraint violated. We suppose this happens due to only this link's congestion condition is changed and other links' are not. We do not miss any intermediate changing point. We then update this link's congestion state. However, it is possible that the net effect of several 
links changing congestion state together gives the same violation. In another word, we may miss changes of the set of congested links. But we believe this case rarely happens. In case it happens, the new updating must give a wrong solution under the new value of $\alpha$. We can tell this by checking the feasible constraints.

Whenever we find we are wrong, we set right to be the current value of $\alpha$ and left to be the value of $\alpha$ of the last iteration, recover the last update and continue to find the next correct update.

Another technique is related to how to choose the starting $\alpha$. In Section 4.2.2, we start from $\alpha=0$. At the starting point, we assume all links are non congested. The equation set $E$ is consisted of linear independent equations. We can get $\lambda_{j}=\alpha W_{j} / P_{j}$ directly. Using the capacity constraint, $\sum_{j \in i} \lambda_{j} \leq C_{i}$. we have

$$
\alpha \leq \frac{C_{i}}{\sum_{j \in i}\left(W_{j} / P_{j}\right)}
$$

The maximum $\alpha$ that satisfies the conditions will be the starting point of $\alpha$. We do not need to increase $\alpha$ from zero.

\subsection{How to Solve the Equations in $E(\alpha)$}

The set of equations in $E(\alpha)$ is a set of nonlinear equations. As a matter of fact, there are no good, general methods for solving systems of more than one nonlinear equations [5]. Finding a root for a set of nonlinear equations which have $\mathrm{N}$ unknowns is to find points which are common to $\mathrm{N}$ unrelated zero-contour hyper-surfaces(the $N$ equations). Each equation is of dimension $N-1$ in a $N$ dimension space. The numerical methods for solving such equations usually involve iterations and matrix operations. The convergence is always a problem. Newton-Raphson Method is the simplest multidimensional root finding method. We borrowed the code from book [5] for the equation solving part.

Given the right set of congested links, Newton-Raphson method converges very well while solving the set of equations in $E$. However, sometimes, we may introduce some redundant constraints. If the load of some links are highly dependent, it is very possible 


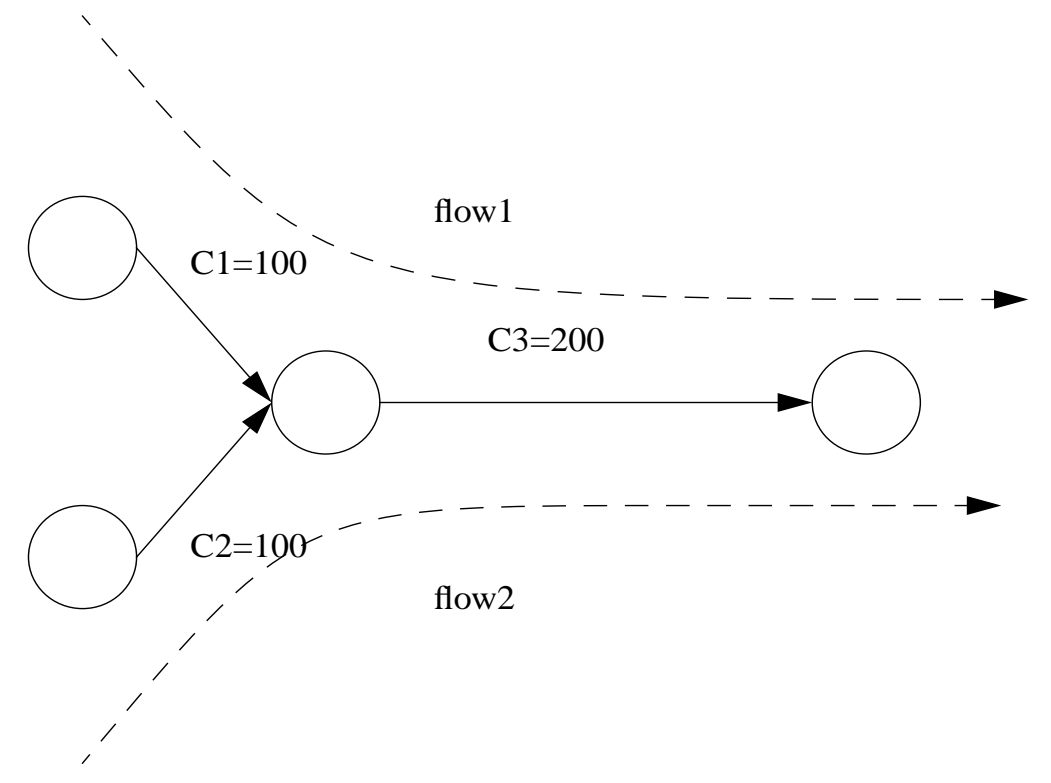

Figure 5-1: A Redundant Constrain: $\left(\lambda_{1}+\lambda_{2} \leq C_{3}\right)$

when all of them are fully loaded, the packets are only backlogged at the first fully loaded link they cross. In this case, the equation has no deterministic answer. In Figure 5-1, when link 1 and 2 are both congested, link 3 is also fully loaded. But packets can not be backlogged at link 3. However, if when we check for the constraints, the loads of all of the three exceed their capacities by a value less than $\epsilon$ under the new value of $\alpha$, based on the first criteria, we will insert them all into Cong and add the capacity constraints for $C_{1}$, $C_{2}$ and $C_{3}$ into $E$. If we directly use Newton-Raphson method, the method will report a singular matrix. Since such a case does not happen very frequently, we do not change the updating algorithm. Instead, if when we solve the equations, this case happens, we need to be conservative. We then add the constraint functions into $E$ one by one. Because we only have one way traffic, the network can be represented as an acyclic graph. We can add the capacity constraint into $E$ in the order flows across the links. This order can be achieved by a topology sort. In the graph, routers are nodes and links are directed edges connecting nodes. We sort the nodes in an order that links always point from lower number nodes to higher number nodes. We insert the link who has a less from-node number than those who has a higher one first. In this way, if a redundant constraint is added, we can detect it immediately. Since packets will be first queued up at the upstream links, we know the newly added constraint must be redundant. Finally, we will delete all redundant constraints 
and correctly solve the equations. At the same time, we can correct the set of congested links set and delete the redundant links. We will mark those links as redundant ones. In the next iteration, we will never choose them as a candidate for congested links.

\subsection{Performance Analysis}

The major cost of the model is the equation solving part. If $E$ contains $k$ valid equations, the time needed to solve the equations is $O\left(\frac{1}{3} k^{3}\right)$. As the number of congested links can not exceed the number of flows, $k$ is at most $2 n$ (Remember $n$ denotes the number of flows.). The positive part of this number is that it is not related to the scale of the networks. Only the number of flows we want to study matters. Unlike the simulation, the cost of solving the equations is not increased with the increase of the number of links a flow across.

The number of iterations used to identify the congested links depends on the network's configurations and the total load. In general cases, a link's congestion state will stay stable within a large range of load, which means, with the increase of $\alpha$, a link does not switch from congestion to non-congestion and vice versa very rapidly. As the algorithm is to try to detect all switching points, if there are not many of them, the number of iterations is moderate then. We believe in reality, cases such as redundant constraints and misjudged congested links due to improper incrementing of $\alpha$ will happen infrequently. Our implementation always tries the aggressive strategy first. That is, when there are several links overloaded or underloaded within a small range, we update the congestion states of all of them simultaneously; when there is only one link overloaded or underloaded, we update its congestion state immediately. In the first case, it is possible to introduce redundant constraints to the equation set. In the second case, our judgment could be wrong if the increment of $\alpha$ is too large and some intermediate switching points are missed. In case either of these happens, we switch to a corresponding backup method. We either update links' congestion states one by one in their topological order or reduce the increment of $\alpha$. The advantage of this strategy is that if the pathological cases do not happen, it runs efficiently. The disadvantage is that it complicates the implementation and in the worst case, it increases the computation cost. 
If we want to further speed up the model, we can change the code of the equation solving part to some optimized solver package. As in this thesis, we focus on the idea more than the technique, we did not try this method. Our goal is to show the validity of the model. We developed the algorithm and the implementation in order to test the model. We believe there are other ways to find a feasible solution for the model.

\subsection{Results Comparison with those of $n s$}

We ran the same test nets on $n s$ and compared the results achieved by modeling and simulating. In all cases, the model gives very accurate results. It is hard to compare the efficiency of the model and the simulation by $n s$ because $n s$ is implemented via Tcl interface, which makes it slower.

\subsubsection{A Brief Introduction of a Test Net}

Here we give one typical test result. The testing net is based on a network topology generated by the software gtitm. It is developed by Ken Calvert and Ellen Zegura, and is available from http://www.cc.gatech.edu/projects/gtitm/gt-itm. The net is hand edited for our need.

The net has 109 links and 110 nodes. We have 21 flows running simultaneously. Each flow has a window size of 20. The test net contains 5 domains. Each domain contains 3 local nets. Links connecting the end user to the subnet are of capacity $8.0 \mathrm{Mb}$. Links connecting intra-localnet routers are of capacity $1.6 \mathrm{Mb}$. Links connecting inter-localnet routers are of capacity $2.4 \mathrm{Mb}$. And links connecting inter-domain routers are of capacity $3.2 \mathrm{Mb}$.

\subsubsection{Results}

The results comparison for queue length is shown in Table 5.1 (Only the congested links are listed.). The results for rates comparison are shown in Table 5.2.

On a Pentium 166MHz computer with Free-BSD OS, it roughly takes the model $0.11 \mathrm{sec}$ to compute the results. For $n s$, we design the simulating time to be 100 seconds. It takes 


\begin{tabular}{|c|c|c|}
\hline link index & $N_{i}$ (model) & $N_{i}(n s)$ \\
\hline 3 & 35.029629 & 34.348 \\
\hline 13 & 52.052593 & 50.7922 \\
\hline 18 & 60.631794 & 58.7596 \\
\hline 65 & 13.021252 & 12.7054 \\
\hline 95 & 27.147820 & 26.8213 \\
\hline 96 & 23.433334 & 22.7227 \\
\hline
\end{tabular}

Table 5.1: Results Comparison: Queue Lengths

\begin{tabular}{|c|c|c|}
\hline flow index & $\lambda_{j}$ (model) & $\lambda_{j}(n s)$ \\
\hline 0 & 78.759331 & 78.06 \\
\hline 1 & 73.871986 & 73.16 \\
\hline 2 & 49.111660 & 49.35 \\
\hline 3 & 49.443676 & 49.53 \\
\hline 4 & 48.813347 & 49.47 \\
\hline 5 & 64.375900 & 63.95 \\
\hline 6 & 70.238083 & 69.72 \\
\hline 7 & 53.623520 & 53.08 \\
\hline 8 & 48.080269 & 47.53 \\
\hline 9 & 67.871315 & 67.48 \\
\hline 10 & 66.501160 & 66.57 \\
\hline 11 & 63.299942 & 63.43 \\
\hline 12 & 71.256714 & 71.92 \\
\hline 13 & 91.414665 & 90.53 \\
\hline 14 & 47.384415 & 47.2 \\
\hline 15 & 161.200943 & 161.55 \\
\hline 16 & 41.864876 & 41.49 \\
\hline 17 & 56.379658 & 56.49 \\
\hline 18 & 100.000000 & 99.93 \\
\hline 19 & 46.004486 & 45.58 \\
\hline 20 & 55.750984 & 56.01 \\
\hline & & \\
\hline
\end{tabular}

Table 5.2: Results Comparison: Average rates of flows 
$n s$ about 65 seconds. 


\section{Chapter 6}

\section{Application Results}

This Chapter discusses the model's application. Since in the real routers, buffer sizes are limited, when a router's buffer overflows, packets will get dropped. TCP will adjust its window size correspondingly. In this Chapter, we assume links are using RED (the Random Early Detection) [6] dropping mechanism. We simulate TCP with RED links by applying this model. In Section 6.1, a brief introduction of RED is listed. In Section 6.2, we discuss the assumptions our simulation depends on. In the next two sections, we discuss the simulating methods and results.

\subsection{RED}

RED is a congestion avoidance mechanism in packet-switched networks. The router monitors the average queue length. If the average queue length exceeds a low threshold, min $_{t h}$, the router notifies the connections of early congestion by either dropping or marking every incoming packet according to a probability. The probability is calculated based on the average queue length. When the average queue length exceeds a maximum threshold, $\max _{t h}$, all incoming packets are either dropped or marked. RED reduces the bias against bursty traffics and avoids the global synchronization of many TCP connections cutting their window sizes by half. 


\subsection{Assumptions}

In the assumptions in Chapter 3, we require each flow's window size to be fixed. However, in a net with RED links, a TCP flow adjusts its window size according to the congestion condition inside the net. Thus the load of the network changes dynamically. The assumption about steady state loses effect. We exploit the ideas of event driven and time driven simulation to simulate the dynamic changes. Still, we ignore the detailed implementation of TCP and RED. Our simple protocol does not consider the slow start, multiple drops and time-out. The parameters we are interested in is the long term average rate a flow could get. We assume a flow's window size is determined by its congestion window. A flow opens its window size by one per round trip time if it does not suffer a packet drop. If a flow suffers at least one packet drop, it cuts its window by half. We implemented two different methods, the event driven simulation and the time driven simulation.

\subsection{The Event Driven Simulation}

\subsubsection{The Simulating Method}

In our simulation, an event refers to a flow's behavior. Either a flow increasing its window size or decreasing its window size is counted as an event. When an event happens, we calculate the current queue length at each router assuming the net with each flow at its current window size has achieved steady state. We use the queue length computed from the model as an approximation of the average queue length a RED router computes. We run RED algorithm based on the current queue length. Since in each round trip time, a flow has a window size of packets distributed across every link along its path, a window size of packets of this flow will pass the RED filter at each congested links. If any of them get dropped, we schedule the next event after the flow's current round trip time with its window cut by half. If none of them get dropped, we schedule the next event after the flow's current round trip time with its window increased by one.

The following is the pseudo-code for the event handler. The part of the code for simulating RED is based on Floyd's paper [6]. The original RED algorithm described in this 
paper keeps a variable count, which is the number of unmarked packets that have arrived since the last marked packet. The dropping probability of a packet is related to count. The variable count is to help marking packets more uniformly. In our model, because we do not trace each packet individually, we can not mark packets in the order of their arrivals. Thus, the variable count does not appear in our code. Other variables such as $\max _{t h}, \min _{t h}$ and $\max _{p}$ have the same meanings as those in that paper.

Argument: current event for flow $j$

Initialization: $\operatorname{mark}=0$

Compute the current queue lengths and flows' rates based on the current window size of each flow for each link $i$ flow $j$ across

if link $i$ is not congested continue

if $N_{i}$ is between $\left[\min _{t h}, \max _{t h}\right]$

$$
p_{a}=\max _{p}\left(N_{i}-\min _{t h}\right) /\left(\max _{t h}-\min _{t h}\right)
$$

for each packet in the flow $j$ 's window

if this packet is going to be dropped according to $p_{a}$

$$
\operatorname{mark}=1
$$

break

else if $N_{i}>\max _{t h}$

$$
\text { mark }=1
$$

if $\operatorname{mark}==1$

break

if $\operatorname{mark}==1$

cut flow $j$ 's window size by half. If the new window size is less than one, set it to one.

else

increase flow $j$ 's window size by one.

Schedule the next event for this flow in the current round trip time of this flow.

For the queue length computing procedure, we can use the algorithm in Chapter 4 to solve the equations directly. That is, we assume all links are not congested at first and 
we iterate through $\alpha$ until we find the final solution. However, if there is no packet drop during last time's computation, during this time's computation, only one flow's window size is increased by one. In this case, links' congestion states should not vary a lot. The last time's congestion links are probably still congested and the non-congested links are still non-congested. We then try last time's congestion link set directly. If this gives us a solution feasible and satisfying all the other constrains, we are set. If this does not, we invoke the original procedure as a back up.

We simulated the situation that a flow detects a packet drop within a single round trip time. Multiple drops in one round trip time is dealt with in the same way as a single drop. We assume this situation is applicable to the real networks.

\subsubsection{Results}

We tested the model's simulation results with those of ns. We designed two test nets. The first one is the test net introduced in Chap 5. The other one is a high speed net with the same topology but each link's capacity is increased by a factor of 10. Links connecting the end user to the subnet are of capacity $80.0 \mathrm{Mb}$. A link connecting intra-localnet routers is of capacity $16 \mathrm{Mb}$. A link connecting inter-localnet routers is of capacity $24 \mathrm{Mb}$. And a link connecting inter-domain routers is of capacity $32 \mathrm{Mb}$. We also have 21 flows. The results are listed in Figure 6-1 and Figure 6-2.

The parameters for RED are $\min _{t h}=10, \max _{t h}=25, \max _{p}=0.2$. In the simulation of $n s$, we use tcp-reno connections to get fast recovery and fast retransmit.

If we assume the results of $n s$ are accurate, the average error of the results for the low speed net is $6.4 \%$. The one for the high speed net is $9.0 \%$.

We are very interested in the speed of the two methods. In the low speed net test, $n s$ wins. It takes $n s$ about 65 seconds to do a 100 seconds simulation. For the model, it costs about 110 seconds. In a low speed net, each flow can only send a small window size of packets every round trip time. Every state of a packet causes an event. Since the cost of packet by packet event driven simulation is proportional to the number of events, if the total number of events is rather small, $n s$ is not slow. However, in the test of a high speed net, it 


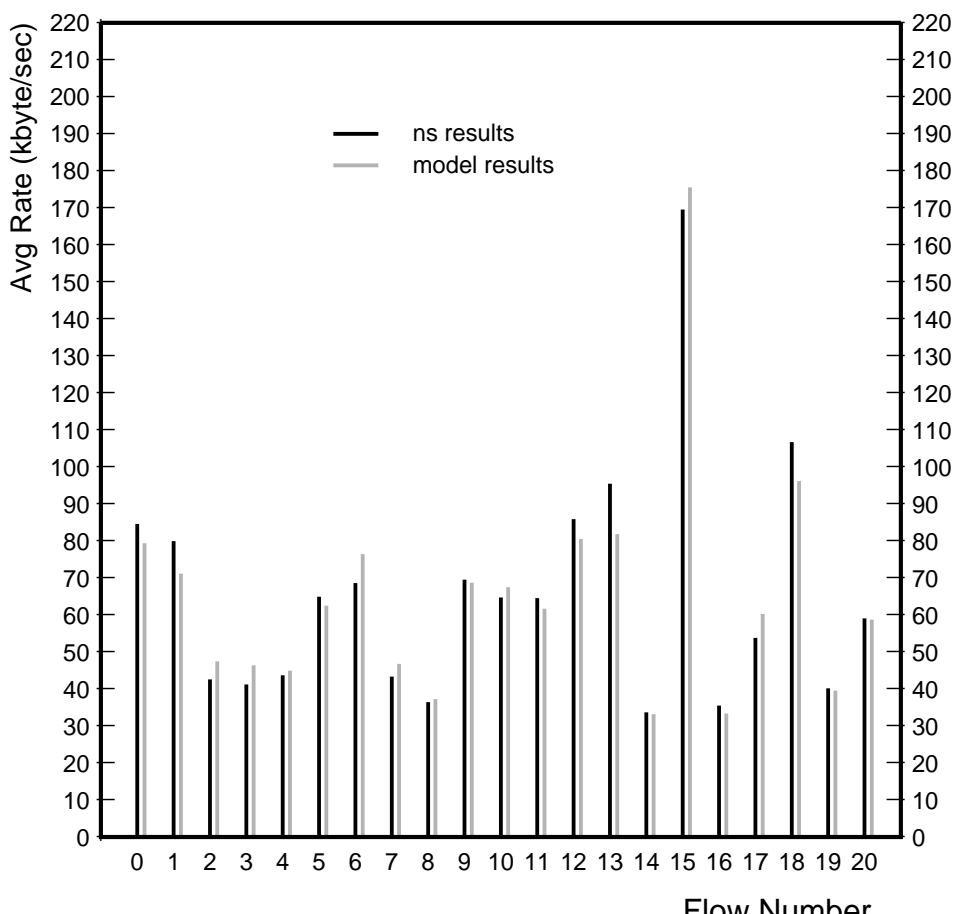

Figure 6-1: Simulation Results of a Low Speed Net

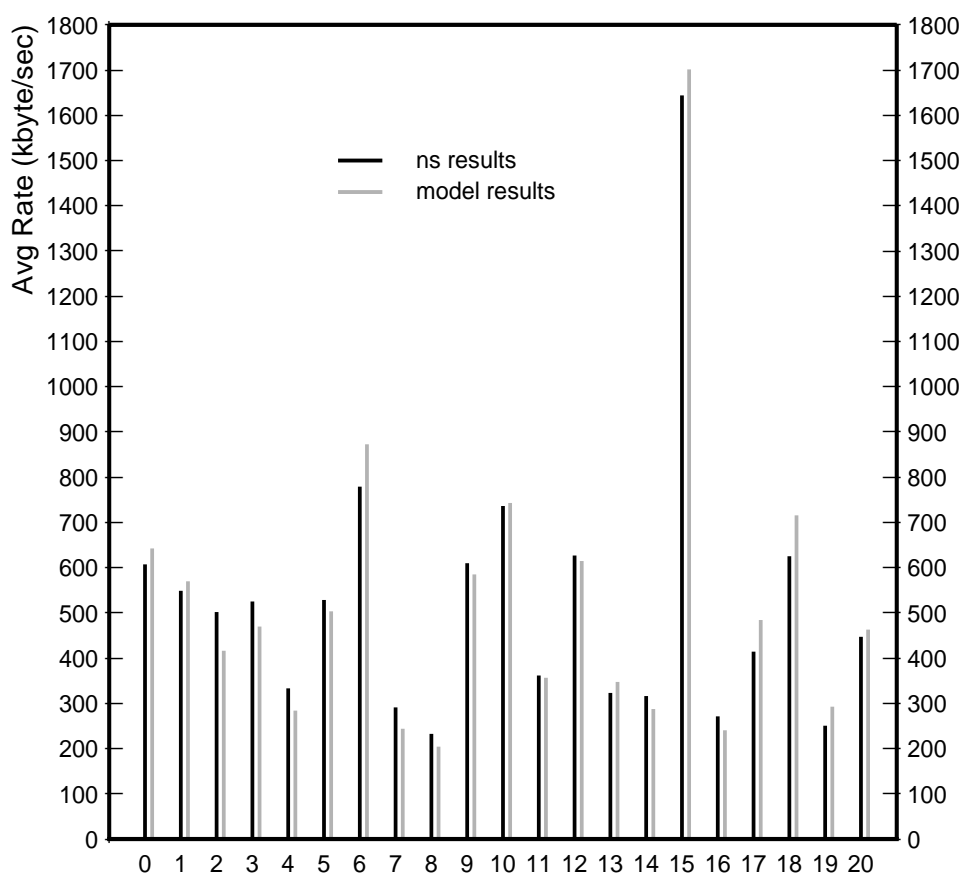

Flow Number

Figure 6-2: Simulation Results of a High Speed Net 
takes $n s$ about 1.3 hours to do a 100 seconds simulation. For the model, the cost is about 11 seconds. The increase of the cost of $n s$ is due to the increase of each flow's window size, which results in the increase of events. A packet by packet event driven simulator has the limit of simulating high speed networks. The decrease of the cost of the model is due to two reasons. One reason is that in the high speed net with the same number of flows, the net is lightly loaded. The number of packet drops is reduced. A flow has more chances to increase its window size by one than it has in a low speed net. Also, it has less chance to cut its window size by half than it has in a low speed net. In this case, links' congestion states do not change dramatically. When an event happens, if we assume the set of congested links is the same as the last event, the assumption is right in most cases. So we do not need to invoke the original $\alpha$ iteration algorithm. The other reason is if a flow with a large window cuts its window size by half, it will greatly reduce the congestion of the network. The network may change from a slightly congested to a non-congested state. For a non-congested or a net with a few congested links, the cost of solving the equations is reduced. As the model's cost is mainly increased with the number of flows, unlike $n s$, the increase of window sizes does not increase its cost. Therefore, the test of a high speed net is much faster than the low one.

\subsection{The Time Driven Simulation}

\subsubsection{The Simulating Method}

In this section, we study a new simulating method. The model is designed to calculate the steady state parameters of a network. It is possible that just after one flow changes its window size, the queue lengths do not change immediately. It may take a short period for the network to reach a new steady state. Based on this idea, we implemented the time driven simulation.

We take a 'snapshot' of the simulated network every delta time. Based on the parameters caught at this time, we decide how each flow will change its window size. We assume each flow will keep its window size within the next delta time. When the next delta time 
passes, we take the snapshot again and decide what will happen in the delta time after this one. Thus the simulation proceeds.

How to choose a proper delta time is a common difficult problem to all time driven simulations. In window based flow control networks, flows change their window sizes according to their own round trip times. It is unlikely that there is a global synchronization that flows change their window sizes if the dropping mechanism is RED. Our simulation is an approximation. Thus the delta time we choose should base on flows' round trip time. The simulating result is sensitive to the value of the delta time. Usually, the delta time should not be less than the largest round trip time among all flows.

Another issue is how to simulate the dropping mechanism of RED. As mentioned above, we choose the same time interval for each flow to change their window sizes. If the time interval is larger than a flow's round trip time, in our simulation, we favor this flow by reducing its packet drop probability since its window size can be cut down at most at the frequency of every interval time, while its window size can still be increased by one every round trip time; on the other hand, if the time interval is shorter than a flow's round trip time, we increase the packet drop probability of this flow since we can cut down its window size every delta time. As flows have different round trip times, no matter how we choose the time interval, such problems exist. Thus, a short round trip flow is likely to have a larger rate share than it should have and vice versa. To compensate for that, we drop a flow's packet according to its rate share of a congested link's capacity and we only let the packets in the current queue pass RED filters. Since the number of a flow's backlogged packets at a congested link is proportional to its share of the capacity ${ }^{1}$, if we drop every single packet according to the rate share of the flow which the packet belongs to, the net probability of a flow getting a packet drop at each delta time is proportional to the square of its rate share. This is different from RED. In RED, when the queue length is between the minimum and the maximum thresholds, every single packet from any flow has the same dropping probability. So the probability of a flow getting a packet drop is roughly linear to its rate. We also tested several other dropping mechanism. Here we only list the

\footnotetext{
${ }^{1}$ If the link is congested, it is transmitting packets all the time. In the steady state, the more backlogged packets a flow has at this link, a more rate share it could achieve.
} 
implementation and results about the above dropping method.

The following pseudo-code is for deciding flows' window sizes within the next delta time.

at time $t$

if $t>$ the total simulating time, stop

for each flow $j$

mark $=0$

for each link $i$ flow $j$ across

if link $i$ is not congested

continue

if $N_{i}$ is between $\left[\min _{t h}, \max _{t h}\right]$

$p_{a}=\max _{p} \times \frac{\lambda_{j}}{C_{i}}$

for each flow $j$ 's packet in the current queue of link $i$

if this packet is going to be dropped according to $p_{a}$

$\operatorname{mark}=1$

break

else if $N_{i}>\max _{t h}$

mark $=1$

if $\operatorname{mark}==1$

break

if $\operatorname{mark}==1$

cut flow $j$ 's window size by half. If the new window size

is less than one, set it to one.

else

increase flow $j$ 's window size by $\frac{\Delta t}{r t t_{j}}$.

$t=t+\Delta t$

\subsubsection{Results}

Figure 6-3 are test results when $\Delta t=0.30 \mathrm{~s}$ for a low speed net. We chose such a time interval because the static round trip time of the test net is ranged from $0.080 \mathrm{~s}$ to $0.26 \mathrm{~s}$. The 


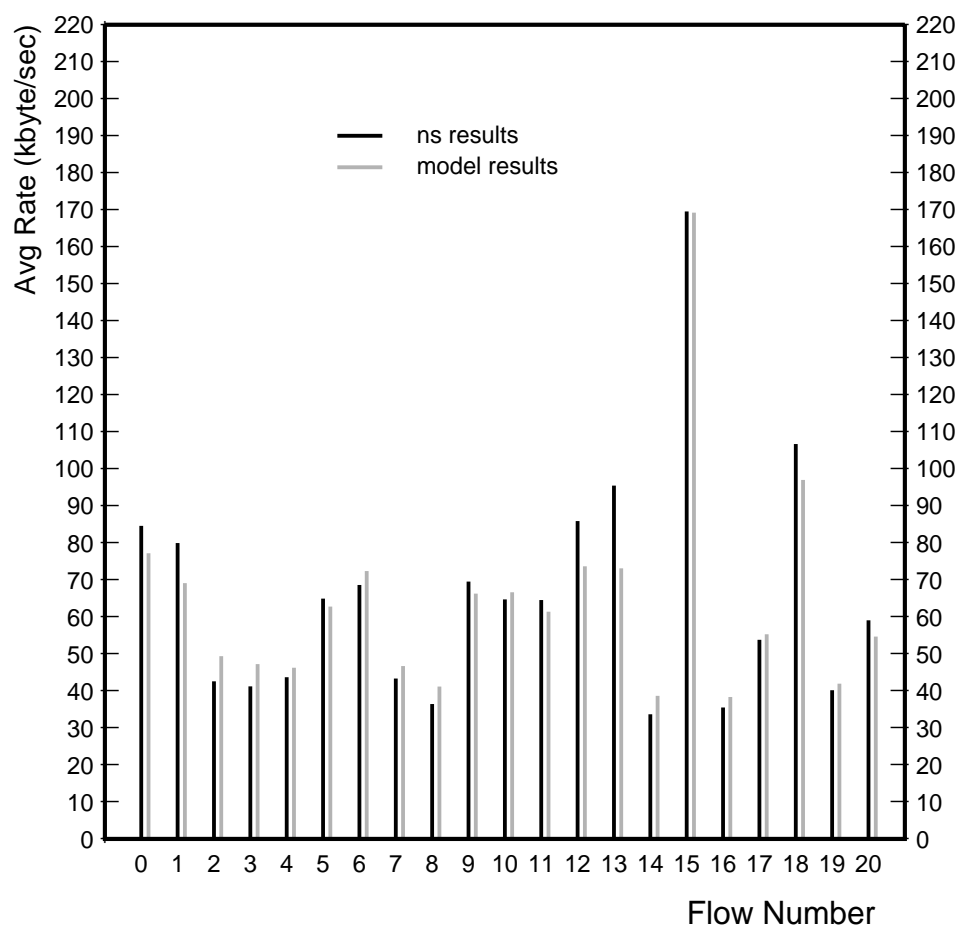

Figure 6-3: Simulation Results of a Low Speed Net: $\Delta t=0.30 \mathrm{~s}$

net is heavily loaded. The real maximum round trip time is around 0.30 s. The simulated time is also 100 seconds. The average running time is 18 seconds. The average error is $8.83 \%$. Figure 6-4 shows the results for simulating a high speed net. $\Delta t=0.25 \mathrm{~s}$. Since the capacities of links are increased by 10 times, the maximum static round trip delay is reduced to $0.24 \mathrm{~s}$. The queuing delay is also reduced. So, we chose a smaller $\Delta t$. The average running time for a 100 seconds simulation is 1.6 seconds. The average error is $6.61 \%$.

Figure 6-5 and 6-6 are test results when $\Delta t=0.50 \mathrm{~s}$. For the low speed net, the running time is nearly 9 seconds. The average error is $8.93 \%$. For the high speed net, the running time is nearly 0.9 second. The average error is $9.84 \%$. We can see when the time interval $\Delta t$ is increased, the running time is decreased correspondingly. In our simulation mechanism, we miss more packets drops if $\Delta t$ is increased. We favor the short round trip delay flows. They increase their window sizes faster than they should. Thus long round trip delay flows get less transmitting rates than they should. The effect can be seen obviously from Figure 6- 


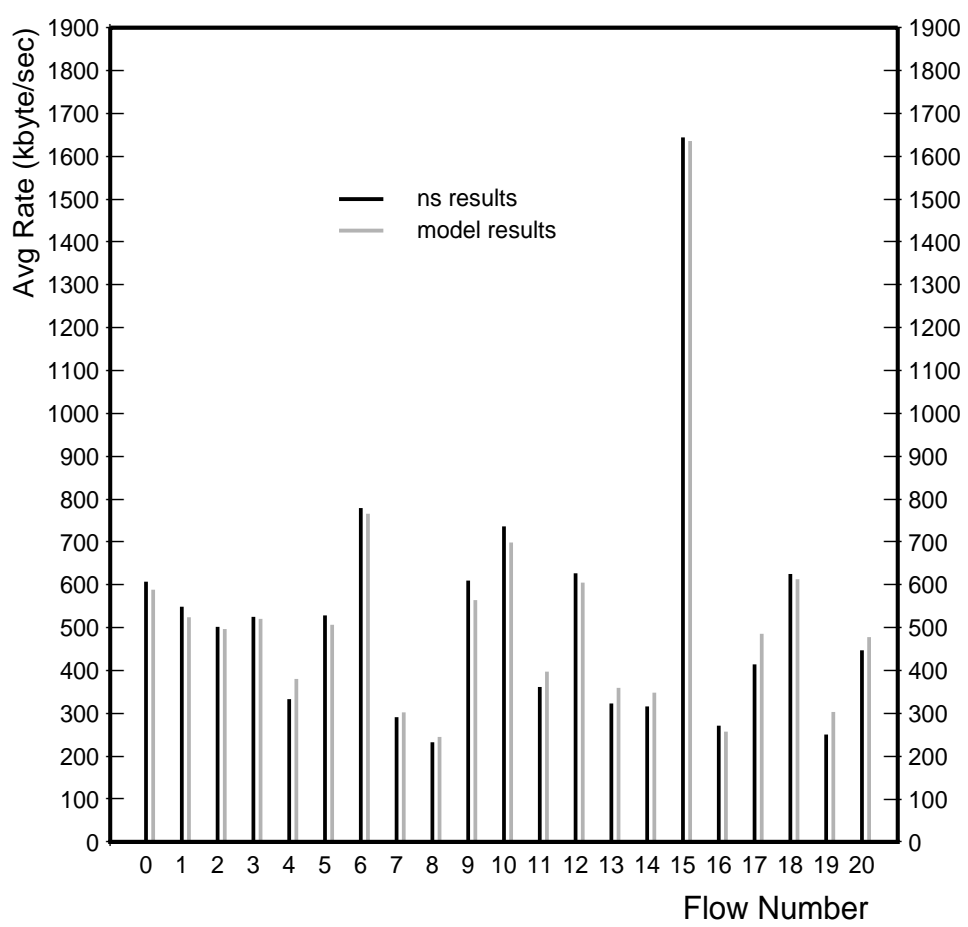

Figure 6-4: Simulation Results of a High Speed Net: $\Delta t=0.25 \mathrm{~s}$

4 and 6-6. Flows have high rates in Figure 6-4 are usually short round trip delay flows. They have higher rates in Figure 6-6. On the contrary, flows have low rates in Figure 6-4 have lower rates in Figure 6-6. 


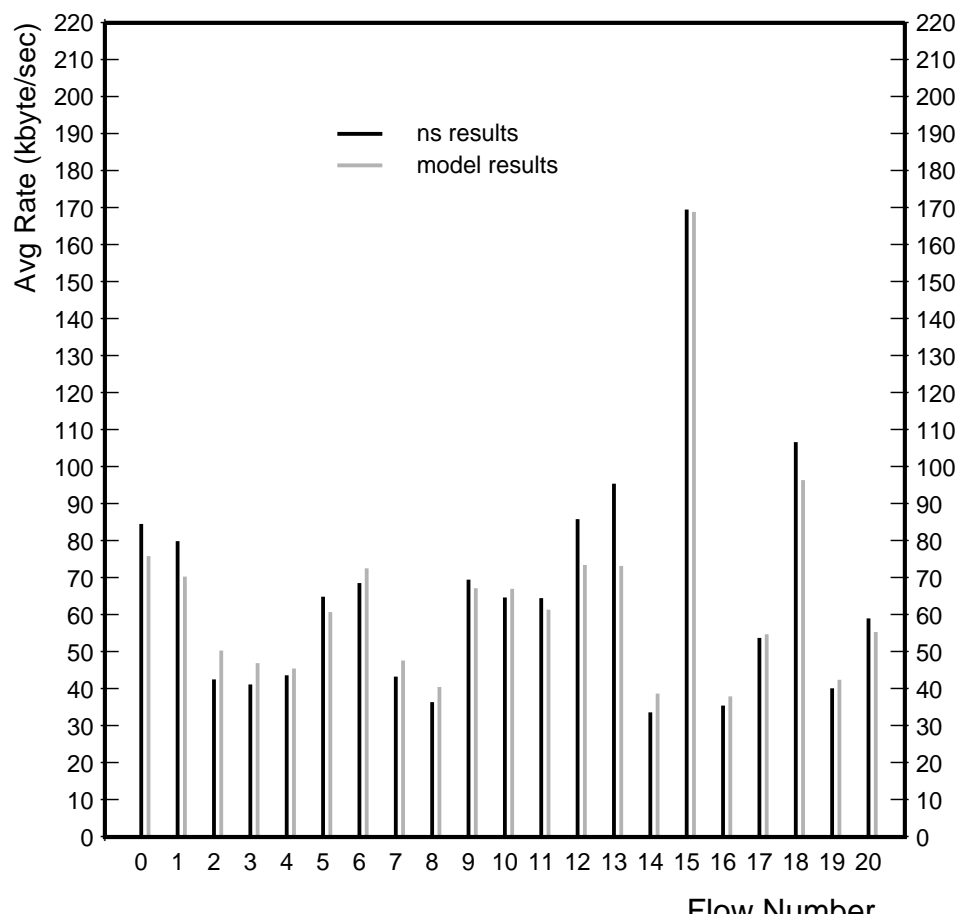

Figure 6-5: Simulation Results of a Low Speed Net: $\Delta t=0.50 \mathrm{~s}$

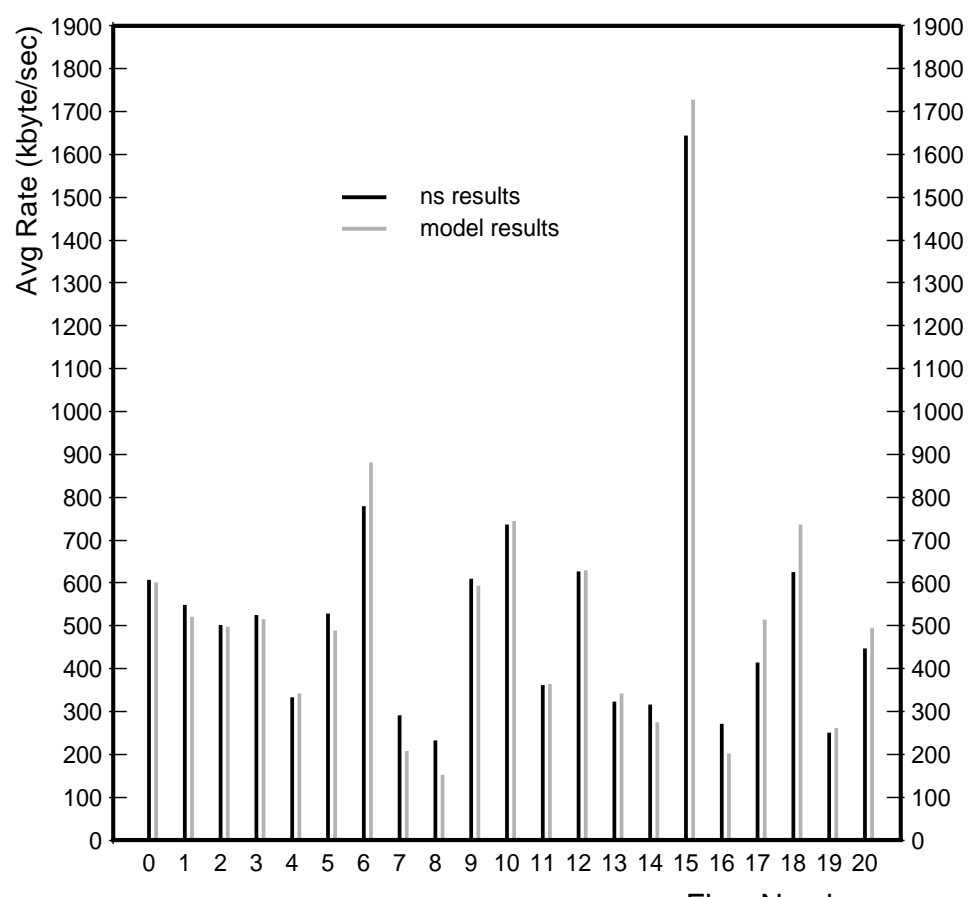

Flow Number

Figure 6-6: Simulation Results of a High Speed Net: $\Delta t=0.50 \mathrm{~s}$ 


\section{Chapter 7}

\section{Summary and Future Work}

We briefly summarize the results from the thesis work and point out some remained questions.

\subsection{Summary}

This thesis introduces a model for studying the steady state of window based flow control packet-switched networks. We construct the model from the observation of TCP's steady state behavior. The model includes the feature of window based flow control mechanism and feasible link capacity constraints. The test results show that the model is a correct abstraction of the real window flow control packet-switched network. We further apply this model to simulate window flow control networks with RED routers. We compared the results from the model and those from $n s$. We do not mean the model can replace the simulation. Simulation is a more accurate way to obtain dynamic state information of networks. However, if we want to get a quick estimation of the long term average statistics, the model's results should be sufficient.

\subsection{Future Work}

There are several remained questions. First of all, the algorithm for finding a solution of the model needs further improvement. We are interested in digging more information from 
the network in order to simplify the model. The only hard problem is how to identify the congested links when each flow's window size is given. We are not sure whether we can achieve that without doing iterations of solving non-linear equations.

The second question is about the testing of the models. The lack of a standard benchmark makes the testing very hard. Even with the help of the software gtiitm, generating a moderate large testing net with flows is a painful task. We should really pay some efforts to develop a set of testing tools to accompany the wide use of simulation.

The third one is how to develop more applications of the model. We chose RED because RED routers drop packets based on the average queue length, which is close to the queue lengths the model computes. We want to find out whether the model can be used to simulate other dropping mechanism.

We hope future work can find out good solutions for the above problems. 


\section{Bibliography}

[1] Dimitri Bertsekas and Robert Gallager. Data Networks. Prentice-Hall, Inc, 1992.

[2] http://netweb.usc.edu/vint/. VINT: Virtual InterNetwork Testbed.

[3] http://www nrg.ee.lbl.gov/ns/. ns version 1 - LBNL Network Simulator.

[4] V. Jacobson. Congestion Avoidance and Control. In Proceedings of the ACM SIGCOMM'88, August 1988.

[5] William H. Press, Saul A. Teukolsky, William T. Vetterling, and Brian P. Flannery. Numerical Recipes in C. the Press Syndicate of the University of Cambridge, 1992.

[6] Floyd S. and Jacobson V. Random Early Detection gateways for Congestion Avoidance. IEEE/ACM Transactions on Networking, 1(4), August 1993.

[7] Anlu Yan and Wei-Bo Gong. Fluid Simulation for High Speed Networks. In Proceedings of the 15th International Teletraffic Congress, June 1997. 